\title{
Understanding the role of silicon oxide shell in oxide-assisted SiNWs growth
}

\author{
S. Q. Wu, ${ }^{1,2, *}$ C. Z. Wang, ${ }^{2}$ Z. Z. Zhu, ${ }^{1}$ and K. M. $\mathrm{Ho}^{2}$ \\ ${ }^{1}$ Department of Physics, Institute of Theoretical Physics and Astrophysics, Xiamen University, \\ Xiamen 361005, P. R. China \\ ${ }^{2}$ Ames Laboratory, U.S. DOE and Iowa State University, Ames, Iowa 50011, USA
}

\begin{abstract}
The role of silicon oxide shell in oxide-assisted SiNWs growth is studied by performing $a b$ initio molecular dynamics simulations on the structural and dynamical properties of the interface between crystalline $\mathrm{Si}(111)$ surface and disorder $\mathrm{SiO}$ thin film. $\mathrm{Si}$ atoms in the $\mathrm{SiO}$ film tends to aggregate into the vicinity of the $\mathrm{Si}(111) / \mathrm{SiO}$ interface. In addition, the diffusion of $\mathrm{Si}$ atoms at the interface is anisotropic - the diffusion along the interface is several times faster than that perpendicular to the interface. The segregation and anisotropic diffusion of Si atoms at the $\mathrm{Si}(111) / \mathrm{SiO}$ interface shed interesting light into the mechanism of oxide-assisted silicon nanowire growth.
\end{abstract}

Keywords: Growth mechanism; Anisotropic diffusion; Silicon nanowire; Oxide-assisted growth; Ab initio molecular dynamics

\footnotetext{
* Corresponding author.

Email address:wsq@xmu.edu.cn
} 


\section{Introduction}

While considerable progress in understanding the atomic and electronic structures of $\mathrm{Si} / \mathrm{SiO}_{2}$ interfaces has been achieved in the last decade $[1,2]$, very few studies have been devoted to silicon suboxides $\mathrm{Si}_{x} \mathrm{O}(2.0>x>0.5)[3,4]$ and $\mathrm{Si} / \mathrm{Si}_{x} \mathrm{O}$ interfaces [5]. Recently, silicon suboxides $\mathrm{Si}_{x} \mathrm{O}$, and $\mathrm{Si} / \mathrm{Si}_{x} \mathrm{O}$ interfaces have been shown to play an important role in the process of oxide-assisted growth (OAG) of silicon nanowires (SiNWs) [6-14] and the performance of $\mathrm{Si} / \mathrm{Si}_{x} \mathrm{O}$ composite anodes in $\mathrm{Li}$-ion batteries $[12,15,16]$. Experiments $[7,8,10,12-14]$ have showed that decomposition of the $\mathrm{Si}$ oxide vapor phase $(\mathrm{SiO})$ resulted in precipitation of $\mathrm{Si}$ nanoparticles and crystalline SiNWs surrounded by silicon oxide shells. It is believed that the structure of the $\mathrm{Si}$ oxide shell and the diffusion of the $\mathrm{Si}$ atoms at the $\mathrm{Si} / \mathrm{Si}_{x} \mathrm{O}$ interface significantly influence the nucleation and growth of the SiNWs $[4,8]$, but the detailed mechanism and many fundamental issues remain elusive.

\section{Methodology}

In this paper, we study the structural properties and dynamical behaviors of solid-liquid $\mathrm{Si}(111) / \mathrm{SiO}$ interface at the atomistic level using ab initio molecular dynamics (AIMD) simulations. We show that $\mathrm{Si}$ atoms aggregate in the vicinity of the $\mathrm{Si}(111) / \mathrm{SiO}$ interface and diffuse much fast along the interface than perpendicular to the interface. The segregation and anisotropic diffusion of $\mathrm{Si}$ atoms at the solid-liquid $\mathrm{Si}(111) / \mathrm{SiO}$ interface shed interesting light into the mechanism of oxide-assisted silicon nanowire growth. 
The $\mathrm{Si}(111)-\mathrm{SiO}$ interface was initially generated by attaching a disordered $\mathrm{SiO}$ film obtained by AIMD simulations to a $\mathrm{Si}(111)$ substrate, to simulate the interfacial system between the crystalline SiNWs and the surrounded silicon oxide shells. AIMD simulations were performed within the density functional theory (DFT) with the projector augmented wave (PAW) method $[17,18]$ to account for the electron-ion interaction, as implemented in VASP [19-21]. The exchange-correlation energy functional is treated within the generalized gradient approximation [22]. The wave functions were expanded in a plane-wave basis with an energy cutoff of $500 \mathrm{eV}$. Only the $\Gamma$ point in the Brillouin zone is used for k-point sampling. The crystalline $\operatorname{Si}(111)$ substrate together with the passivated $\mathrm{H}$ atoms at the bottom are fixed during the high temperature simulations in order to avoid the melting of the substrate at the high temperature (to simulate the crystalline $\mathrm{Si}$ core of SiNWs from OAG) while both $\mathrm{Si}$ and $\mathrm{O}$ atoms in the $\mathrm{SiO}$ film are allowed to move according to Newton's equations of motions with forces from quantum mechanical DFT calculations. The interface structure in our simulations contains 100 atoms in the $\mathrm{SiO}$ film region, and $54 \mathrm{Si}$ atoms and $9 \mathrm{H}$ atom in the substrate. After the interface structure has been optimized we reheat the sample to $2500 \mathrm{~K}$ to study the diffusion behaviors. High temperature simulation is used here in order to speed up the diffusion process due to the time limitation in the AIMD simulations. The statistics of the structural and dynamical properties of the interface system were evaluated over a $30 \mathrm{ps}$ (after a thermalization run of $12 \mathrm{ps}$ ) with a time step of 3 fs.

\section{Results and discussion}




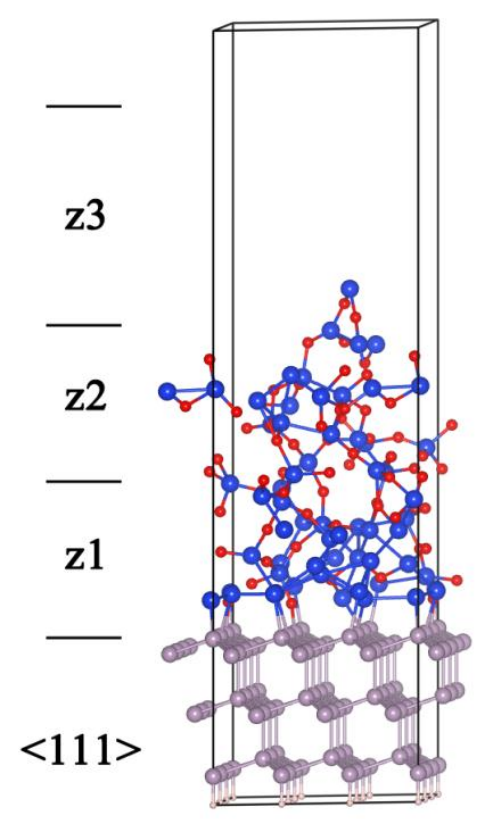

Fig. 1. (Color online). Representative snapshot for $\mathrm{Si}(111) / \mathrm{SiO}$ interface. Purple (light grey): $\mathrm{Si}$ atoms in crystal substrate; blue (dark grey, lager): $\mathrm{Si}$ atoms in $\mathrm{SiO}$ layer; red (dark grey, smaller): $\mathrm{O}$ atoms.

A representative structure of the $\mathrm{Si}(111) / \mathrm{SiO}$ interface is shown in Fig. 1. To have a better understanding on the simulation results, the $\mathrm{SiO}$ film at the interface is divided into three regions $z 1, z 2$, and $z 3$ according to their vertical distance from the interface. The thickness of $z 1$ and $z 2$ are chose to be $8 \AA$, i.e. 0.2 in the scaled coordinates. To eliminate the effect of the bottom of slab on the diffusion of $\mathrm{Si}$ or $\mathrm{O}$ atoms, $z 3$ is only counted up to 0.9 in the scaled coordinates. The results of the MD simulations are quantified in Fig. 2(a), which shows the density profiles of the $\mathrm{Si}$ atoms together with the average $\mathrm{Si}$ over $\mathrm{O}$ ratios (i.e., $x$ in $\mathrm{Si}_{x} \mathrm{O}$ ) at different regions of the interface. Compositional segregation of $\mathrm{Si}$ from the $\mathrm{SiO}$ film to the interfacial area $(z 1)$ is observed. $\mathrm{Si}$ atoms in the $\mathrm{SiO}$ film tends to aggregate into the vicinity of the $\mathrm{Si}(111) / \mathrm{SiO}$ 
interface with a $\mathrm{Si} / \mathrm{O}$ ratio of 1.54 in the $z 1$ region. The $\mathrm{Si} / \mathrm{O}$ ratio is only 0.74 and 0.83 in the $z 2$ and $z 3$ regions respectively.

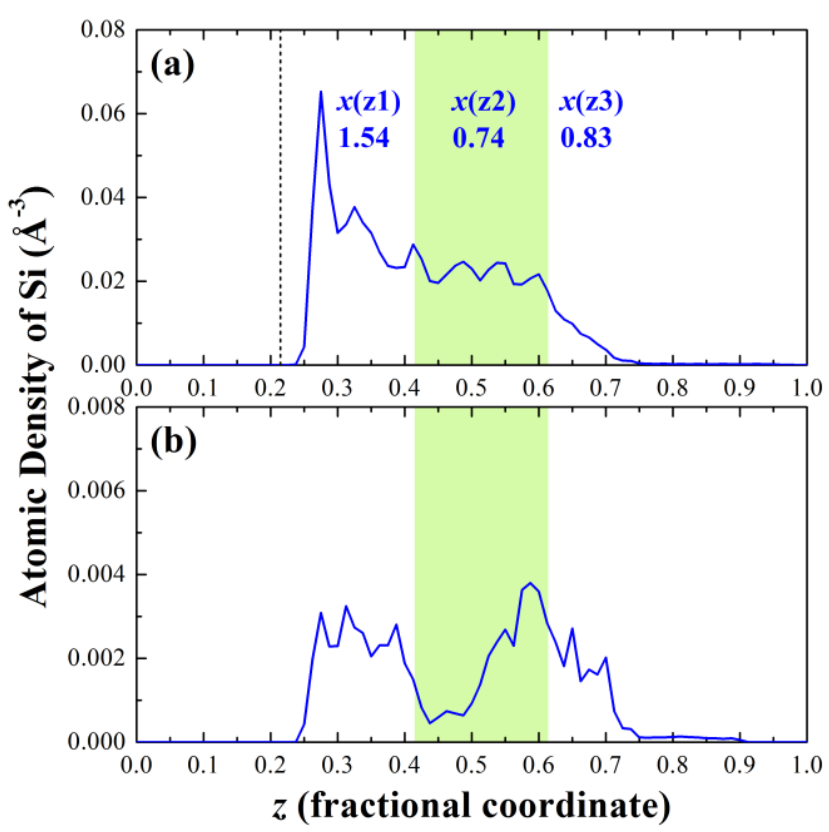

Fig. 2. (Color online). Density profiles of (a) $\mathrm{Si}$ atoms and (b) fast (top 10\%) $\mathrm{Si}$ atoms in $\mathrm{SiO}$ layer in $\mathrm{Si}(111): \mathrm{SiO}$. The $z$-coordinate is along a direction perpendicular to the interface plane.

To investigate the dynamical behaviors of the $\mathrm{Si}$ atoms at the interface, the mean square displacements (MSD) of the $\mathrm{Si}$ atoms in the $\mathrm{SiO}$ film at the non-Gaussian time interval $\left(t^{*}\right)$ [23] at the temperature of $2500 \mathrm{~K}$ are calculated. The non-Gaussian time corresponds to the time at which the non-Gaussian parameter distribution reaches its maximum value [23, 24]. This time interval is long enough that it goes beyond the ballistic motion regime, but short enough that the initial environment of the atom has important effects on the motion of the atom within this time interval thus produces the most heterogeneous dynamical behavior [25]. The density profiles of 
the fast Si atoms at different distances from the interface are shown in Fig. 2(b). Most of the fast $\mathrm{Si}$ atoms are located in the regions of $z 1$ and $z 3$, therefore the distribution of the fast diffusing $\mathrm{Si}$ atoms exhibits a valley in the $z 2$ zone as one can see from Fig. 2(b). Si atoms diffuse faster at the interface is the consequence of the oxygen-poor environment due to the compositional segregation.

We can also use the trajectories from the AIMD simulations to study the diffusion behavior of the $\mathrm{Si}$ atoms in different regions of the interface. Fig. 3 gives some representative trajectories of the $\mathrm{Si}$ atoms at $\mathrm{Si}(111) / \mathrm{SiO}$ interface. The figures in the left side of Fig. 3 show the projection of trajectories on the $x y$ plane, while the right ones are the projection on the $x z$ plane. In these figures, the dark and grey balls show the top bilayer of $\operatorname{Si}(111)$ surface and the $z 2$ region is highlighted in grey. Different colors in the trajectories correspond to Si atoms that have different numbers of nearest oxygen neighbor (NBR) as indicated at the different simulation time. The results show that $\mathrm{Si}$ atoms in the $z 1$ region exhibit fast diffusion (Fig.3 (a) and (b)), while those in the $z 2$ region oscillate locally (Fig. 3 (c) and (d)). This can also be easily seen from Fig. 3 (e) and (f). When a $\mathrm{Si}$ atom goes into the $z 3$ region, the diffusion can be very fast due to the vaporization of $\mathrm{SiO}$ molecules, as shown in Fig. $3(\mathrm{~g})$ and $(\mathrm{h})$. In our previous study, we have shown that $\mathrm{Si}$ atoms with more O neighbors in molten Si-rich oxides have lower diffusivity [4]. This "oxygen slowing-down" effect [4] can also be seen in the $z 1$ and $z 2$ regions of the $\mathrm{Si}(111) / \mathrm{SiO}$ interface studied in this paper. Besides the $\mathrm{SiO}$ molecule, some $\mathrm{Si}$ atoms with 2 oxygen neighbors in $z 3$ also show fast diffusion. This is due to the fact that there are two types of 
$\mathrm{SiO}$ vaporization: single-molecular $(\mathrm{SiO})$ and bimolecular $\left(\mathrm{Si}_{2} \mathrm{O}_{2}\right)$ vaporization.
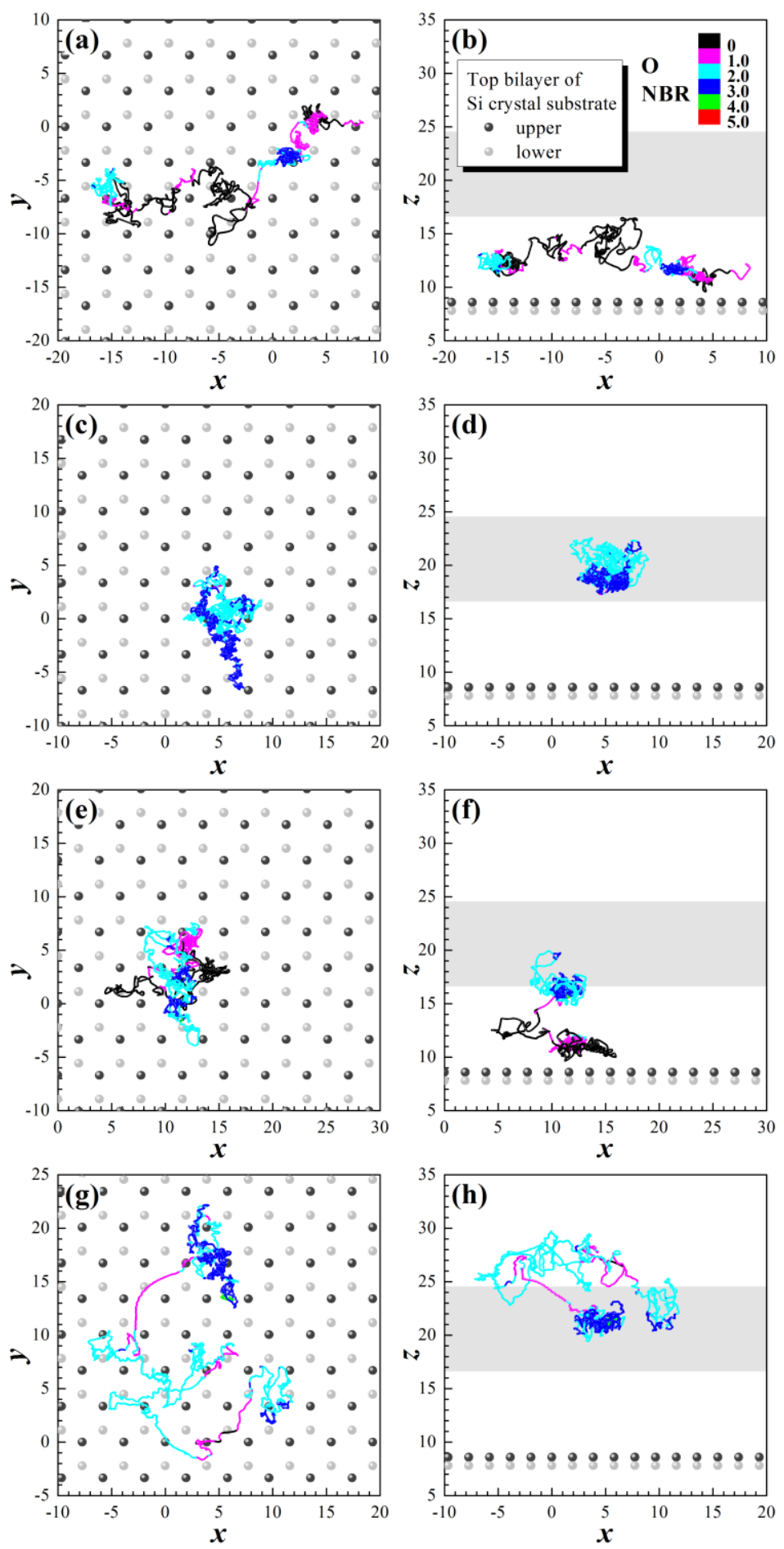

Fig. 3. (Color online). Representative trajectories of $\mathrm{Si}$ atoms. 
Table I. Ratios of the MSD at the time interval of non-Gaussian time and the diffusion constant parallel to interface to those perpendicular to interface. $\left\langle x^{2}+y^{2}\right\rangle_{z 1}$ and $D_{x y, z 1}$ are the MSD values and diffusion constant of $\mathrm{Si}$ atoms in the $x y$-plane in the $z 1$ region. $\langle z 2\rangle_{z 1}$ is the MSD of Si atoms along the interface normal direction in the $z 1$ region. $\langle z 2\rangle_{z 1+z 2}$ and $D_{z, z 1+z 2}$ are the MSD values and diffusion constant of $\mathrm{Si}$ atoms along the $\mathrm{z}$-direction in the $\mathrm{z} 1$ and $\mathrm{z} 2$ regions.

\begin{tabular}{cccc}
\hline$\frac{\left\langle x^{2}+y^{2}\right\rangle_{z 1}}{\left.<z^{2}\right\rangle_{z 1}}$ & $\frac{\left\langle x^{2}+y^{2}\right\rangle_{z 1}}{\left.<z^{2}\right\rangle_{z 1+z 2}}$ & $\frac{D_{x y, z 1}}{D_{z, z 1}}$ & $\frac{D_{x y, z 1}}{D_{z, z 1+z 2}}$ \\
\hline 19.5 & 13.9 & 15.2 & 15.1 \\
\hline
\end{tabular}

We found diffusion of the $\mathrm{Si}$ atoms in the $\mathrm{Si}(111) / \mathrm{SiO}$ interface is strongly anisotropic with the diffusion parallel to the interface 15-20 times faster than the diffusion perpendicular to the interface. This diffusion anisotropic behavior can be clearly seen from the ratios of the MSD at the time interval of non-Gaussian time and the diffusion constant parallel to the interface to those perpendicular to the interface as listed in Table I. Here, the diffusion of Si atoms along the $z$-direction in $z 1$ and $z 2$ regions can be understood as the diffusion of $\mathrm{Si}$ atoms crossing the silicon oxide shell in oxide-assisted SiNWs growth; while the $x y$-plane diffusion of $\mathrm{Si}$ atoms in $z 1$ region can be understood as the transport of $\mathrm{Si}$ atoms along the interface. These ratios as listed in Table I are between 14 and 20.

The aggregation and the anisotropic diffusion of $\mathrm{Si}$ at the interface would play an important 
role in the oxide-assisted growth of SiNWs. First of all, the fast diffusion along the silicon-rich interface (the $z 1$ region) due to the Si aggregation can facilitate fast growth of the NW along the axial direction (NW direction). Secondly, as we will discuss in the following, the unsaturated $\mathrm{SiO}_{y}(y<2)$ instead of saturated $\mathrm{SiO}_{2}$ shell around the $\mathrm{NW}$ would allow continued incorporation of $\mathrm{SiO}$ molecular from the gas phase into the interface and ensure the continued SiNW growth through the aggregation and fast interface diffusion mechanism discussed above.

According to our simulations, the value of $y$ in $\mathrm{SiO}_{y}$ film in the $z 2$ and $z 3$ regions is around 1.4, which is much less than 2 . This result suggests that the silicon oxide shell of SiNWs from OAG is a $\mathrm{Si}$-rich oxide layer rather than $\mathrm{SiO}_{2}$. This conclusion reinforces our view in the previous work on the Si-rich oxide melts [4]. In fact, most of the studies in the literature proposed that the outer shell of SiNWs from OAG is the silicon dioxide simply based on the reaction, $\mathrm{SiO}($ gas $) \rightarrow \mathrm{Si}($ solid $)+\mathrm{SiO}_{2}$ (solid $)$. Our simulation results indicate that the thermal equilibrium of this reaction may not be easy to reach during the SiNW growth process because the dynamics of $\mathrm{SiO}_{2}$ is very slow [4] even at high temperatures above its melting point. It is interesting to note that there has been experimental evidence that if the SiNWs were exposed to the air for long enough time and then taken back to the growth zone, they would not grow any more [26]. This observation is consistent with our findings that the outer silicon oxide shell around the NW during the growth is sub-stoichiometric $\mathrm{SiO}_{y}(y<2)$ rather than $\mathrm{SiO}_{2}$.

Recent experiment [12] clearly indicated that the silicon oxide shell of SiNWs is not $\mathrm{SiO}_{2}$. 
However, K.W. Lin et al. [12] also stated that the stoichiometry of Si and O in the shell could not be determined due to variation of atomic ratio of $\mathrm{Si}$ and $\mathrm{O}$ at different samples, and there is variation of atomic ratios at different shell positions in a sample. It can be understood based on our results (see Fig. 2a) where the average $\mathrm{Si}$ over $\mathrm{O}$ ratios (i.e., $x$ in $\mathrm{Si}_{x} \mathrm{O}$ ) are varied at different regions of the interface. Considering the excellent electrochemical performances of $\mathrm{Si}_{-} \mathrm{SiO}_{y}$ core-shell NWs as an anode for lithium-ion batteries and the important role that the $\mathrm{Si} / \mathrm{SiO}_{y}$ shell plays, i.e. it can efficiently prevent the volume expansion of the $\mathrm{Si}[12], \mathrm{Si}_{/} / \mathrm{Si}_{x} \mathrm{O}$ interfaces in the SiNWs deserve further experimental and theoretical investigations.

Finally we would like to point out that since the most favorite growth direction for SiNWs prepared by the oxide-assisted growth is the $\langle 112\rangle$ direction and the surfaces of the SiNWs along this direction are mostly bounded by the $\{111\}$ facets [27], it is reasonable to use the $\mathrm{Si}(111) / \mathrm{SiO}$ interface system to understand the oxide-assisted SiNW growth mechanism. The above analysis and discussion based on ab initio MD simulations allow us to gain insight into how the dynamical behaviors are related to the fast growth in oxide-assisted SiNWs growth.

\section{Conclusions}

In conclusion, $a b$ initio molecular dynamics simulations have been performed on a solid-liquid silicon/silicon-oxide interface system to study the structural and dynamical properties, and to understand the role of silicon oxide shell in oxide-assisted SiNWs growth. Compositional segregation of $\mathrm{Si}$ in $\mathrm{SiO}$ layer is observed in the $\mathrm{Si}(111) / \mathrm{SiO}$ interface. We proposed that the 
silicon oxide shell of $\mathrm{SiNWs}$ from $\mathrm{OAG}$ is $\mathrm{SiO}_{y}(y<2)$ rather than pure $\mathrm{SiO}_{2}$. The diffusion behavior of Si atoms in the interface system reinforces our view of the "oxygen slowing-down" effect as found in the Si-rich oxide melts. In addition, the diffusion anisotropy is found for $\mathrm{Si}$ atoms in the interface which plays an important role in oxide-assisted SiNWs growth.

\section{Acknowledgements}

Work at Ames Laboratory was supported by the US Department of Energy, Basic Energy

Sciences, Division of Materials Science and Engineering including a grant of computer time at the National Energy Research Supercomputing Centre (NERSC) in Berkeley, under Contract No. DE-AC02-07CH11358. SQW also acknowledges financial support from the National Natural Science Foundation of China (No. 11004165).

\section{References}

[1] A. Pasquarello, M. S. Hybertsen, R. Car, Nature 396 (1998) 58-60.

[2] K. Hirose, H. Nohira, K. Azuma, T. Hattori, Progress in Surface Science 82 (2007) 3-54

[3] S.M. Schnurre, J. Gröbner, R. Schmid-Fetzer, J. Non-Cryst. Solids 336 (2004) 1.

[4] S.Q. Wu, C.Z. Wang, Z.Z. Zhu, K.M. Ho, Appl. Phys. Lett. 96 (2010) 043121.

[5] K. Yin, Y. Zhao, X. Wang, L. Liu, G. Xue, S.-T. Lee, and M. Shao, Appl. Phys. Lett. 104, 033110 (2014).

[6] N. Wang, Y.F. Zhang, Y.H. Tang, C.S. Lee, S.T. Lee, Appl. Phys. Lett. 73 (1998) 3902.

[7] N. Wang, Y.H. Tang, Y.F. Zhang, C.S. Lee, S.T. Lee, Phys. Rev. B 58 (1998) R16024. 
[8] N. Wang, Y.H. Tang, Y.F. Zhang, C.S. Lee, I. Bello, S.T. Lee, Chem. Phys. Lett. 299 (1999) 237.

[9] X.D. Xu, Y.C. Wang, Z.F. Liu, R.G. Zhao, Adv. Funct. Mater. 17 (2007) 1729-1734.

[10] N. Wang, Y. Cai, R.Q. Zhang, Mat. Sci. Eng. R 60 (2008) 1.

[11] X.D. Xu, S.B. Li, Y.C. Wang, T.J. Fan, Y.D. Jiang, L. Huang, Q. He, T.H. Ao, Nanoscale Res. Lett. 7 (2012) 243.

[12] K. W. Lim, J.-I. Lee, J. Yang, Y.-K. Kim, H. Y. Jeong, S. Park, and H. S. Shin, ACS Appl. Mater. Interfaces 6, 6340-6345 (2014).

[13] E.G. Barbagiovanni, D.J. Lockwood, P.J. Simpson, and L.V. Goncharova, Appl. Phys. Rev. 1, 011302 (2014).

[14] S.K. Behura, Q. Yang, A. Hirose, O. Jani, I. Mukhopadhyay, J. Mater. Sci. 49, 3592 (2014).

[15] C.M. Park, W. Choi, Y. Hwa, J.H. Kim, G. Jeong, H.J. Sohn, J. Mater. Chem. 20 (2010) 4854-4860.

[16] T. Zhang, J. Gao, H.P. Zhang, L.C. Yang, Y.P. Wu, H.Q. Wu, Electrochem. Commun. 9 (2007) 886-890.

[17] P.E. Blöchl, Phys. Rev. B 50 (1994) 17953.

[18] G. Kresse, D. Joubert, Phys. Rev. B 59 (1999) 1758.

[19] G. Kresse, J. Furthmüller, Comput. Mater. Sci. 6 (1996) 15.

[20] G. Kresse, J. Furthmüller, Phys. Rev. B 54 (1996) 11169.

[21] G. Kresse, J. Non-Cryst. Solids 192-193 (1995) 222.

[22] J.P. Perdew, J.A. Chevary, S.H. Vosko, K.A. Jackson, M.R. Pederson, D.J. Singh, C. 
Fiolhais, Phys. Rev. B 46 (1992) 6671.

[23] W. Kob, and H.C. Anderson, Phys. Rev. E 51, 4626 (1995).

[24] M.Z. Li, C.-Z. Wang, M.I. Mendelev, and K.M. Ho, Phys. Rev. B 77, 184202 (2008).

[25] S.G. Hao, C.Z. Wang, M.J. Kramer, and K.M. Ho, J. Appl. Phys. 107, 053511 (2010).

[26] N. Wang, personal communication.

[27] C.P. Li, C.S. Lee, X.L. Ma, N. Wang, R.Q. Zhang, S.T. Lee, Adv. Mater. 15, 607 (2003). 